\title{
Left atrial dimension and cardiovascular outcomes in patients with and without atrial fibrillation: a systematic review and meta-analysis
}

\author{
Lorin Froehlich, ${ }^{1,2}$ Pascal Meyre (D) ,1,2 Stefanie Aeschbacher (D) , 1,2 Steffen Blum, 1,2 \\ Daniela Djokic, ${ }^{2}$ Michael Kuehne, ${ }^{1,2}$ Stefan Osswald, 1,2 Beat A Kaufmann, 1,2 \\ David Conen ${ }^{2,3}$
}

\begin{abstract}
- Additional material is published online only. To view please visit the journal online (http://dx.doi.org/10.1136/ heartjnl-2019-315174).

'Division of Cardiology, Department of Medicine, University Hospital Basel, Basel, Switzerland

${ }^{2}$ Cardiovascular Research Institute Basel, University Hospital Basel, Basel, Switzerland

${ }^{3}$ Population Health Research Institute, Hamilton, Ontario, Canada
\end{abstract}

\section{Correspondence to} Professor David Conen, Population Health Research Institute, McMaster University, Hamilton, Canada; conend@mcmaster.ca

LF and PM contributed equally.

Received 4 April 2019 Revised 15 July 2019 Accepted 24 July 2019 Published Online First 17 August 2019

\section{Linked}

- http://dx.doi.org/10.1136/ heartjnl-2019-315695

\section{Check for updates}

(C) Author(s) (or their employer(s)) 2019. No commercial re-use. See rights and permissions. Published by BMJ.

To cite: Froehlich L, Meyre $P$, Aeschbacher $S$, et al. Heart 2019;105:1884-1891.

\section{ABSTRACT \\ Objective The prognostic value of left atrial (LA) dimensions may differ between patients with and without atrial fibrillation (AF).}

Methods MEDLINE and EMBASE were searched for studies that investigated the association between LA echocardiographic parameters measured by transthoracic echocardiography and cardiovascular outcomes in patients with or without AF. Data were independently abstracted by two reviewers and pooled using randomeffects meta-analysis. The primary outcome was incident stroke or thromboembolic events. Secondary outcomes were heart failure, all-cause mortality and major adverse cardiac events (MACE).

Results Twenty-three studies of patients with AF (14939 patients) and 68 studies of patients without AF (50 720 patients) in this systematic review. Increasing LA diameter was significantly associated with stroke and thromboembolic events in patients without AF (risk ratio (RR) $1.38,95 \% \mathrm{Cl} 1.02$ to $1.87 ; \mathrm{p}=0.03$ ), but not in patients with AF (RR 1.02, 95\% Cl 0.98 to 1.07; $p=0.27 ; p$ for difference $=0.05)$. Increasing LA diameter index was significantly associated with MACE in patients with $A F(\operatorname{RR~1.13,95\% ~Cl~} 1.09$ to 1.17; $p<0.001)$ and in patients without $A F(R R 2.98,95 \%$ Cl 1.90 to 4.66; $p<0.001$ ), with stronger effects in non-AF populations ( $p$ for difference $<0.001$ ). Greater LA volume index was significantly associated with the risk of MACE in patients with AF (RR 1.01, 95\% CI 1.00 to 1.02; $p=0.03$ ) and in non-AF populations (RR 1.08, 95\% Cl 1.05 to 1.10; $p<0.001)$, the association being stronger in individuals without AF ( $p$ for difference $<0.001$ ).

Conclusions Larger LA parameters were associated with various adverse cardiovascular events. Many of these associations were stronger in individuals without $A F$, highlighting the potential importance of $L A$ myopathy.

\section{INTRODUCTION}

Atrial fibrillation (AF) is the most common cardiac arrhythmia, and its prevalence is expected to further increase over time. ${ }^{12}$ Patients with AF have an increased risk of stroke, heart failure and death. ${ }^{3-5}$

The concept of atrial myopathy has been recently introduced to take into account the fact that patients with atrial disease have an increased risk of stroke and other complications in the absence of clinical
AF. ${ }^{6}$ Potential determinants of atrial myopathy are an increased left atrial (LA) size, atrial wall fibrosis or an increased number of premature atrial contractions. ${ }^{7}$ While studies have shown an increased risk of adverse events in patients with markers of LA myopathy, the strength of the associations has not been systematically compared with that of patients with established AF.

In support of this concept, LA enlargement, usually defined as anterior-posterior LA diameter $\geq 4.0 \mathrm{~cm}$, has been significantly associated with incident $\mathrm{AF}$, stroke and death among individuals without AF. ${ }^{89}$ By contrast, studies including patients with AF not always confirmed the associations with adverse cardiovascular outcomes. ${ }^{10} 11$

Therefore, the objective of this systematic review and meta-analysis was to determine the associations of LA echocardiographic dimensions with the risk of stroke, heart failure, all-cause mortality and major adverse cardiac events among individuals with versus those without AF.

\section{METHODS}

The protocol of this systematic review was registeredwith PROSPERO (CRD42018116166). This study is reported according to the Meta-analysis of Observational Studies in Epidemiology criteria. ${ }^{12}$

\section{Eligibility criteria}

Studies were included if they enrolled patients with $\mathrm{AF}$, patients without $\mathrm{AF}$ or provided separate effect estimates for the two populations. Studies among patients with AF were either exclusively AF studies or had to report an AF prevalence of $>90 \%$. Studies among patients without AF had to explicitly state that they excluded patients with AF or their prevalence of patients with AF was $<10 \%$. We included only longitudinal studies, irrespective of date of publication, language or geographical distribution. Abstracts that met inclusion criteria were included. Cross-sectional or case-control studies, and studies with $<100$ participants were excluded. Eligible studies had to investigate associations between four prespecified LA parameters assessed by transthoracic echocardiography and a predefined set of clinical outcomes. These parameters were LA diameter (LAD), LA diameter indexed to body surface (LADI), LA volume (LAV) and LA volume indexed to body surface (LAVI). The primary outcome 
for this meta-analysis was stroke or thromboembolic events; secondary outcomes were heart failure, all-cause mortality and major adverse cardiac events, defined as any composite of major cardiovascular outcomes.

\section{Literature search methods}

A comprehensive systematic search in MEDLINE and EMBASE was conducted from database inception to 7 July 2018. The search strategy was reviewed by an experienced librarian and is outlined in the online supplementary material, Methods S1. Controlled vocabulary using Medical Subject Heading (MeSH) terms and keywords for AF, echocardiography, LA parameters and cardiovascular outcomes were used for the search. Reference lists of eligible records were subsequently screened, and experts in the field were consulted in order to identify additional studies.

\section{Data collection}

Two independent reviewers (LF and PM) screened titles and abstracts of all studies from the initial search. Full texts of potentially eligible studies were retrieved and reviewed by the same reviewers for definitive inclusion. Reasons for exclusion were noted. Disagreements were resolved through discussion or third party arbitration (DC). Study authors were contacted to request additional information missing from the initial record.

\section{Data extraction}

A standardised case report form was used to extract study-level and patient-level characteristics from each study, including year of publication, study design, population sample, sample size, follow-up duration, average age, sex distribution and concomitant conditions, including coronary artery disease, stroke or transient ischaemic attack, heart failure and history of major bleeding. Extracted data were entered into a Microsoft Access database (Microsoft, Redmond, Washington, USA).

\section{Assessment of study quality}

The methodological quality of included studies was evaluated using a modified version of the Newcastle-Ottawa Scale. ${ }^{13}$ Details of the study quality assessment are presented in online supplementary material. In brief, we rated representativeness of patient population, adjustments for effect estimates, outcome assessment and description of the echocardiographic measurements. Ten to eight points indicated high quality, 7-5 points moderate quality and $<5$ points low study quality.

\section{Statistical analysis}

From each study, we extracted the reported ORs or HRs, and calculated the risk ratios (RRs) with corresponding 95\% CIs for LA echocardiographic parameters and the outcomes indicated above. Data transformations were performed according to the recommendations of the Cochrane Handbook for Systematic Reviews. ${ }^{14}$ For the main analyses, we included studies that used a continuous measure of LA echocardiographic parameters (ie, per unit increase in millimetres or per increase in SD). Studies reporting only on categorical LA measures were assessed in secondary analyses. When more than two categories were reported, we included the RR for the highest compared with the lowest category. For all analyses, we extracted the most extensively adjusted estimate available. Estimates for each outcome were pooled using inverse variance-weighted random-effects models since considerable between-study heterogeneity was expected. ${ }^{15}$ Between-study heterogeneity was assessed using Cochran's Q statistic and quantified by the $\mathrm{I}^{2}$ statistic. $\mathrm{I}^{2}$ values $\geq 50 \%$ were considered to indicate substantial heterogeneity. ${ }^{14}$ All analyses were performed separately in studies with patients with AF and studies with patients without AF. The two individually pooled RRs were then compared using stratified metaanalysis to assess whether the observed differences between the two populations were statistically significant.

To explore between-study heterogeneity, we performed subgroup analyses based on study design, geographical region and publication year, and random-effects meta-regression analyses for sample size, follow-up duration, age and male sex. Subgroup and meta-regression analyses were carried out only if at least 10 studies were available. Evidence for small-study effects were examined by visual inspection of funnel plots and by Egger's test. ${ }^{1617}$ All analyses were performed using RevMan V.5.3 (Cochrane Collaboration) or Stata V.13.0 (StataCorp). A two-tailed $\mathrm{p}$ value $<0.05$ was considered statistically significant.

\section{RESULTS}

\section{Characteristics of included studies}

A total of 23 studies including patients with AF $(n=14936)$ and 68 studies of individuals without AF $(n=50720)$ met inclusion criteria and were included in the meta-analysis (figure 1). Characteristics of included studies are presented in online supplementary tables S2 and S3.

\section{Associations of LAD with cardiovascular outcomes}

LAD was not significantly associated with stroke and thromboembolic events in patients with AF (RR 1.02, 95\% CI 0.98 to $1.07 ; \mathrm{I}^{2}=49 \%$ ), but was significantly associated with this outcome in patients without AF (RR $1.38,95 \%$ CI 1.02 to 1.87 ; $\left.\mathrm{I}^{2}=85 \%\right)(\mathrm{p}$ for difference $=0.05$ ) (table 1 ; figure $2 \mathrm{~A}$ ). In studies that analysed LAD as categorical variable, we found significant associations in patients with AF and those without AF (see online supplementary figure $\mathrm{S} 1$ ). LAD was associated with incident heart failure in patients with AF (RR 1.07, 95\% CI 1.03 to 1.11 ; $\left.\mathrm{I}^{2}=13 \%\right)$ and in patients without AF (RR $1.05,95 \%$ CI 1.03 to $\left.1.07 ; \mathrm{I}^{2}=0 \%\right)(\mathrm{p}$ for difference $=0.31$ ) (figure $2 \mathrm{~B}$ ). Findings were similar in studies where LAD was analysed as a categorical variable (see online supplementary figure S1). LAD was not associated with risk of death in patients with AF (RR 1.05, 95\% CI 0.99 to $\left.1.12 ; \mathrm{I}^{2}=\mathrm{NA}\right)$, but this association was significant in patients without AF (RR 1.29, 95\% CI 1.08 to $1.54 ; \mathrm{I}^{2}=78 \%$ ) ( $\mathrm{p}$ for difference $=0.03$ ) (figure $2 \mathrm{C}$ ). Findings were similar in studies using categorical LAD variables. LAD was associated with MACE in patients with AF (RR 1.74, 95\% CI 1.09 to 2.77; $\mathrm{I}^{2}=\mathrm{NA}$ ) and patients without AF (RR $1.34,95 \%$ CI 1.18 to 1.52 ; $\mathrm{I}^{2}=87 \%$ ) ( $\mathrm{p}$ for difference $=0.28$ ) (figure 2D). One study that used $\mathrm{LAD}$ as categorical variable in patients without AF found a significant association between LAD and MACE.

\section{Associations of LADI with cardiovascular outcomes}

LADI was significantly associated with risk of stroke and thromboembolic events in patients with AF (RR 1.07, 95\% CI 1.04 to $1.10 ; \mathrm{I}^{2}=25 \%$ ) and non-AF populations (RR 1.11, 95\% CI 1.00 to $1.22 ; \mathrm{I}^{2}=\mathrm{NA}$ ) ( $\mathrm{p}$ for difference $=0.49$ ) (table 1 ; figure $3 \mathrm{~A}$ ). Similar findings were observed in studies where LADI was analysed categorically (see online supplementary figure S2). The association of LADI and risk of death was only evaluated using LADI as a categorical variable. In one study of patients with AF, the association was RR $1.28,95 \% \mathrm{CI} 0.97$ to $1.69 ; \mathrm{I}^{2}=\mathrm{NA}$, and in three studies of patients without $\mathrm{AF}$, the association was RR $1.88,95 \%$ CI 1.43 to $2.47 ; \mathrm{I}^{2}=0$; $\mathrm{p}$ for difference $=0.05$ (see online supplementary figure S2). For the relationship of LADI 


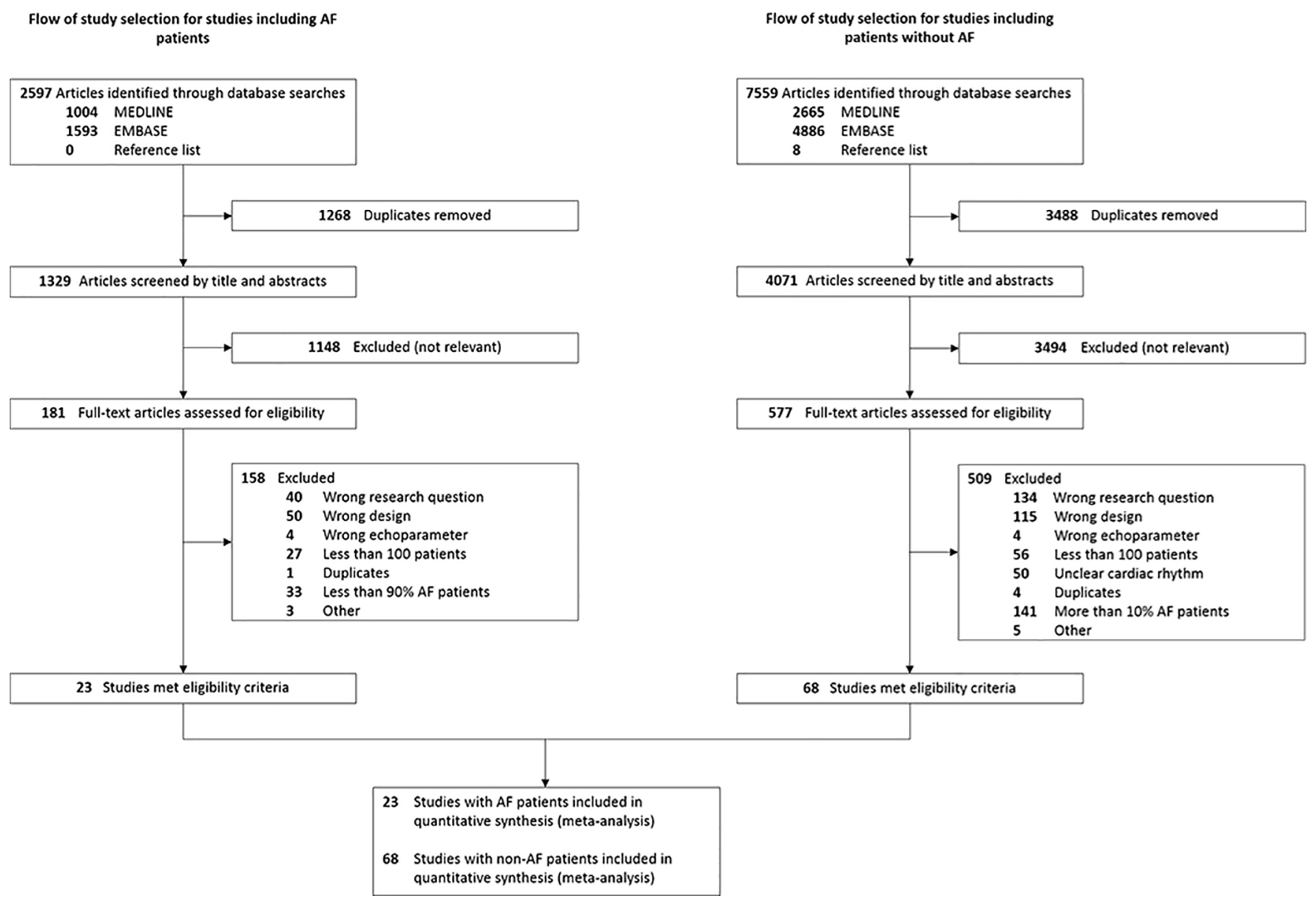

Figure 1 Flow diagrams of selection for studies including patients with atrial fibrillation (AF) and studies without patients with AF.

and risk of MACE, we found a significant association in patients with AF (RR 1.13, 95\% CI 1.09 to $1.17 ; \mathrm{I}^{2}=\mathrm{NA}$ ) and in non-AF populations (RR 2.98, 95\% CI 1.90 to 4.66 ; $\mathrm{I}^{2}=0$ ) (p for difference $<0.001$ ) (figure $3 \mathrm{~B}$ ). LADI as a categorical variable was not associated with MACE in patients without AF (see online supplementary figure S2).

\section{Associations of LAV with cardiovascular outcomes}

LAV was not associated with stroke and thromboembolic events in patients with AF (table 1), but it was associated with the risk of heart failure, death and MACE in patients without AF. These observations were similar in studies that used LAV as a categorical variable (see online supplementary figure S3).

\section{Associations of LAVI with cardiovascular outcomes}

LAVI was not associated with stroke and thromboembolic events in either patients with AF (RR 1.02, 95\% CI 1.00 to $1.04 ; \mathrm{I}^{2}=73$ ) or without $\mathrm{AF}$ (RR 1.22, 95\% CI 0.94 to 1.59 ; $\mathrm{I}^{2}=72 \%$ ) ( $\mathrm{p}$ for difference $=0.17)$ (table 1 ; figure $4 \mathrm{~A})$. Similar results were found in studies that analysed LAVI in a categorical fashion among patients without AF (see online supplementary figure S4). LAVI was associated with incident heart failure in patients without AF (RR $1.07,95 \%$ CI 1.03 to $1.10 ; \mathrm{I}^{2}=75 \%$ ), but not in patients with AF (RR 1.11, 95\% CI 0.95 to 1.29 ; $\mathrm{I}^{2}=89 \%$ ) (p for difference $=0.63$ ) (figure 4B). Five studies in patients without AF that used LAVI as a categorical variable found a RR of 1.77 (95\% CI 1.25 to 2.49 ; $\mathrm{I}^{2}=35 \%$ ) (see online supplementary figure S4). LAVI was associated with death in patients without AF (RR 1.05, 95\% CI 1.02 to $1.07 ; \mathrm{I}^{2}=80 \%$ ), but not in patients with AF (RR
$1.10,95 \%$ CI 0.78 to $1.55 ; \mathrm{I}^{2}=80 \%$ ) ( $\mathrm{p}$ for difference $=0.78$ ) (figure 4C). In studies using categorical LAVI variables among patients without AF, similar findings were observed (see online supplementary figure S4). LAVI was associated with a higher risk of MACE in patients with AF (RR 1.01, 95\% CI 1.00 to 1.02 ; $\left.\mathrm{I}^{2}=0 \%\right)$ and patients without AF (RR $1.08,95 \%$ CI 1.05 to 1.10 ; $\mathrm{I}^{2}=88 \%$ ) ( $\mathrm{p}$ for difference $<0.001$ ) (figure 4D). Findings were similar in studies of patients without AF that analysed LAVI as a categorical variable (see online supplementary figure S4).

\section{Study quality and sensitivity analyses}

Overall study quality of included studies was high (see online supplementary tables S3 and S4). After removing studies of low quality, the main findings of our analyses remained unchanged (see online supplementary table S5).

Subgroup and meta-regression analyses had to be restricted by the limited number of studies available. In studies with patients without AF, differences in the association of LAVI with all-cause death and MACE were observed, with greater risks found in randomised controlled trials (see online supplementary table S6). Meta-regression analyses did not indicate an effect of patient characteristics on the observed associations (see online supplementary table S7).

\section{Assessment of small-study effects}

The assessment of small-study effects was limited by the small number of studies (see online supplementary figures S3 and S4). In studies including patients without AF, the funnel plot revealed some asymmetry and Egger's test showed nominal evidence for 


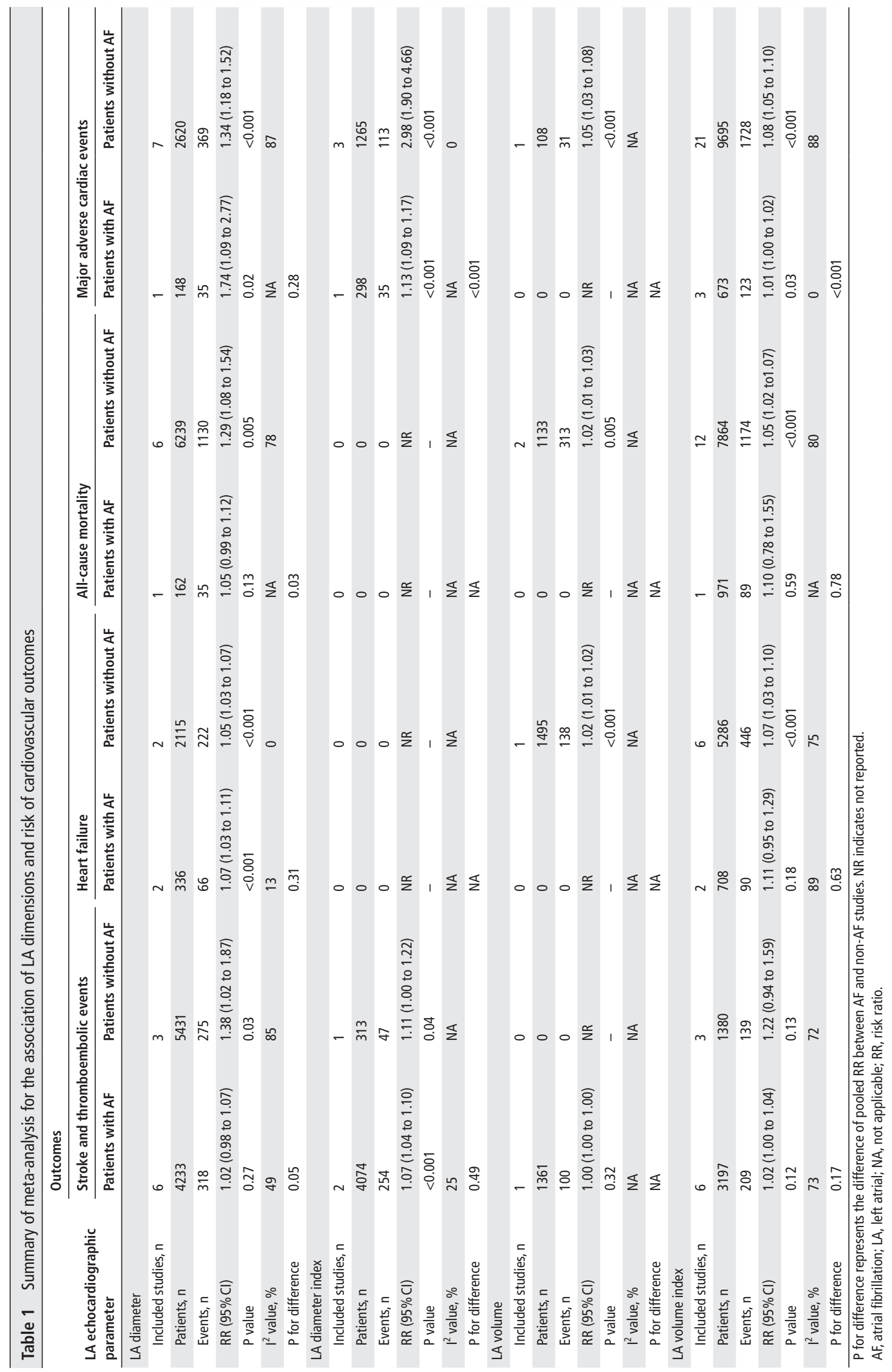


A

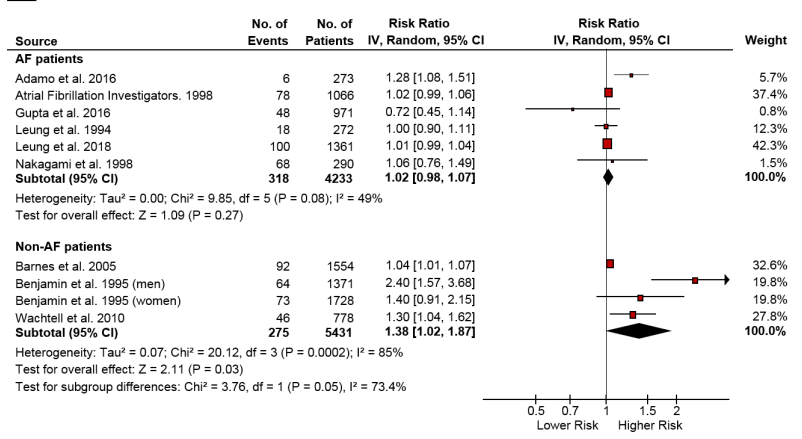

C

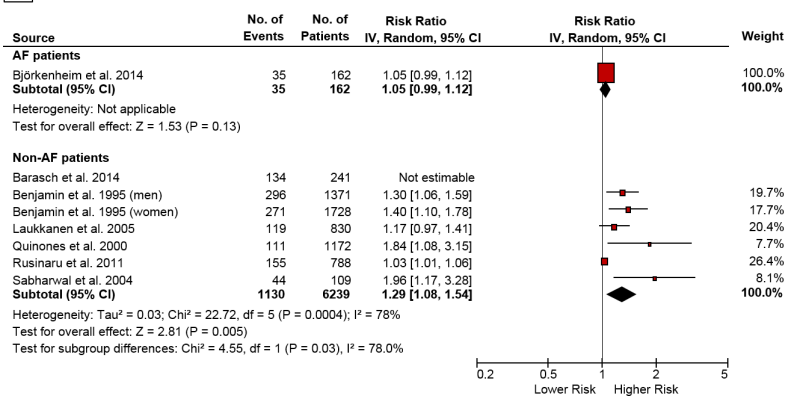

B

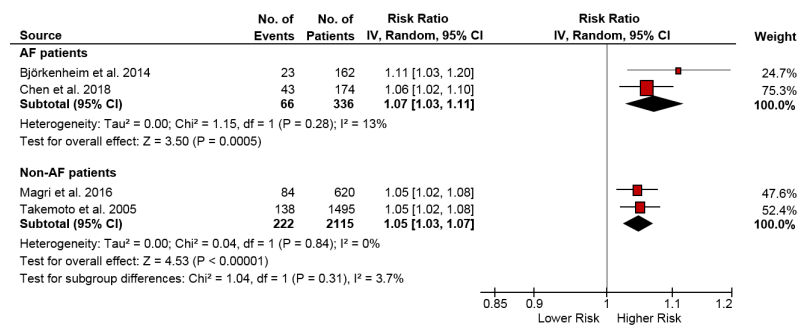

D

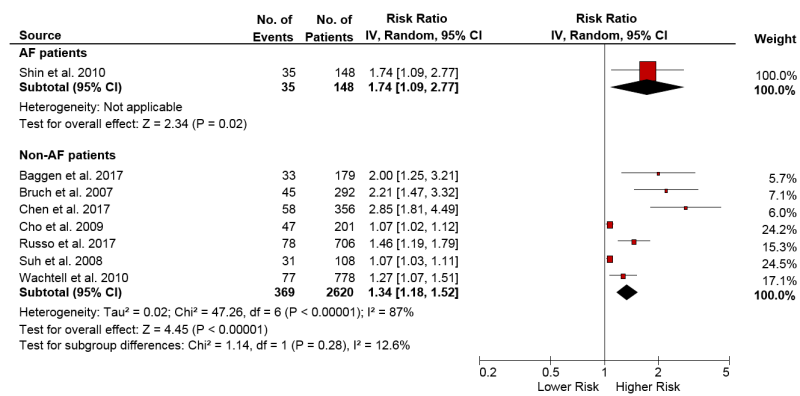

Figure 2 Association of LA diameter and risk of cardiovascular outcomes. Association of LA diameter with incident stroke or thromboembolic events (A), heart failure (B), all-cause mortality (C) and major adverse cardiac events (D). Data are risk ratios with corresponding $95 \%$ Cls from inverse variance-weighted (IV) random-effects models. Meta-analysis results are separately presented as diamonds for studies including patients with AF and studies without patients with AF. Barasch et al only reported a p value of 0.0004 for the association of LA diameter and the risk of death. AF, atrial fibrillation; LA, left atrial.

small-study effects for the association of LAD and the risk of death and MACE (Egger's test $p=0.004$ and $p=0.001$, respectively). There was some evidence of small-study effects for the association of LAVI with MACE, which was confirmed by Egger's test $(\mathrm{p}<0.001)$.

\section{DISCUSSION}

In this large and comprehensive systematic review and metaanalysis of 91 studies including 65659 individuals, at least two important findings emerged. First, while LAD parameters were generally associated with stroke and thromboembolic events, LAV parameters were not. Second, most of the associations differed at least nominally between patients with and without AF, with stronger effects in patients without AF.

Atrial myopathy has recently been proposed as an important contributor to the risk of stroke and other adverse outcomes, independent of the occurrence of $\mathrm{AF}^{18}{ }^{18}$ The classical concept is that AF causes haemodynamic changes which favour clot formation in the LA appendage. These clots may then lead to embolisation in the cerebral circulation and stroke. ${ }^{19}$ Although most risk differences between populations did not reach formal statistical significance, we observed generally stronger associations of LA parameters with stroke or thromboembolism in patients without AF. This finding supports the concept of LA myopathy and may indicate that structural properties of the LA as part of the LA myopathy concept may contribute to thromboembolic complications in the absence of AF.

LA dilation may be a marker of longstanding exposition to cardiovascular risk factors, such as hypertension or obesity, or it may be a marker of overall structural heart disease in patients with coronary artery disease, mitral valve stenosis or left ventricular dysfunction. ${ }^{20} 21$ Left ventricular diastolic dysfunction (LVDD), left ventricular hypertrophy and the resulting increase in LA wall stress are important features of the atrial remodelling

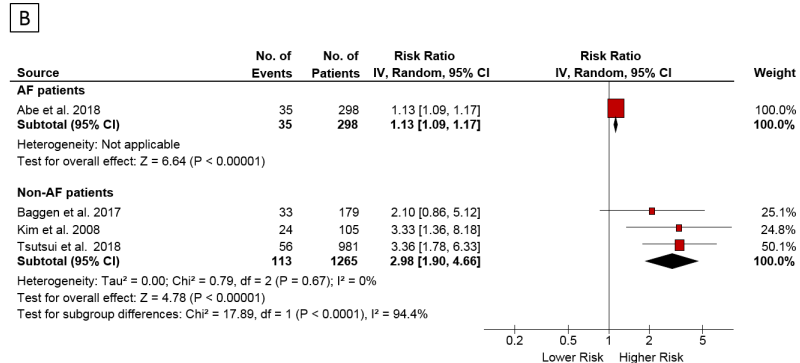

Figure 3 Association of LA diameter index and risk of cardiovascular outcomes. Association of LA diameter index with incident stroke or thromboembolic events (A), and major adverse cardiac events (B). Data are risk ratios with corresponding $95 \%$ Cls from inverse variance-weighted (IV) random-effects models. Meta-analysis results are separately presented as diamonds for studies including patients with AF and studies without patients with $A F$. AF, atrial fibrillation; LA, left atrial. 
A

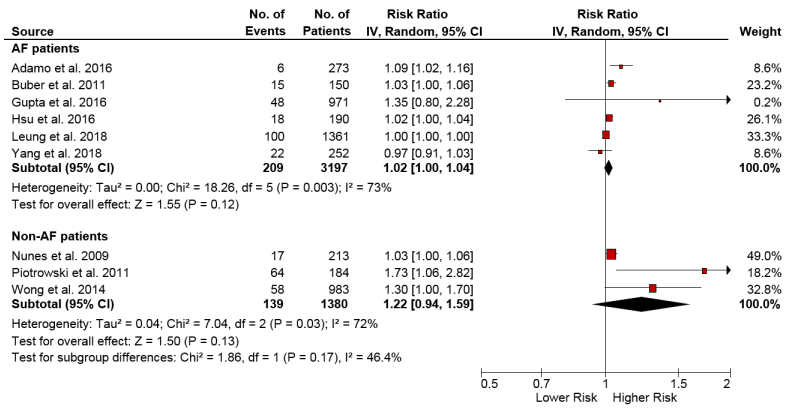

C

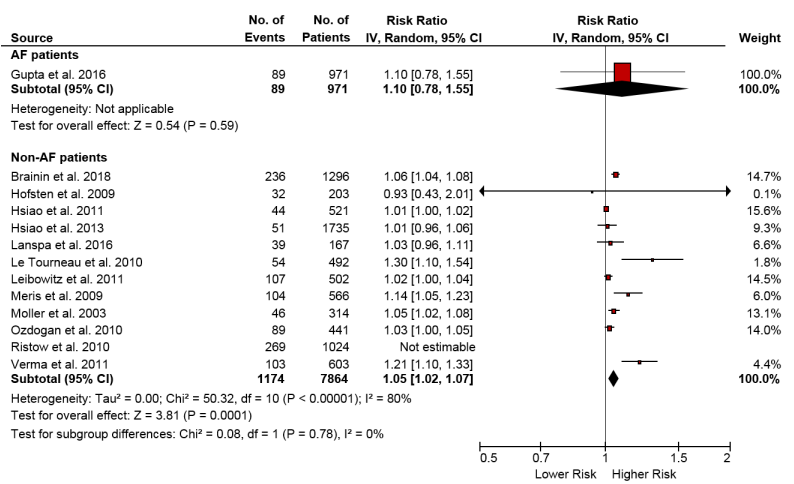

B

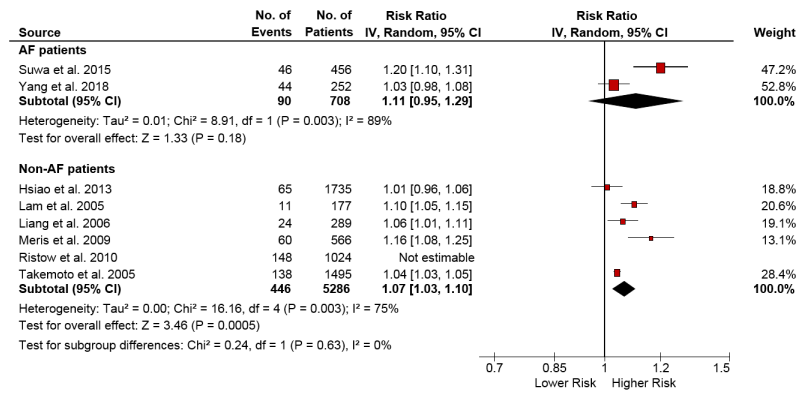

$D$

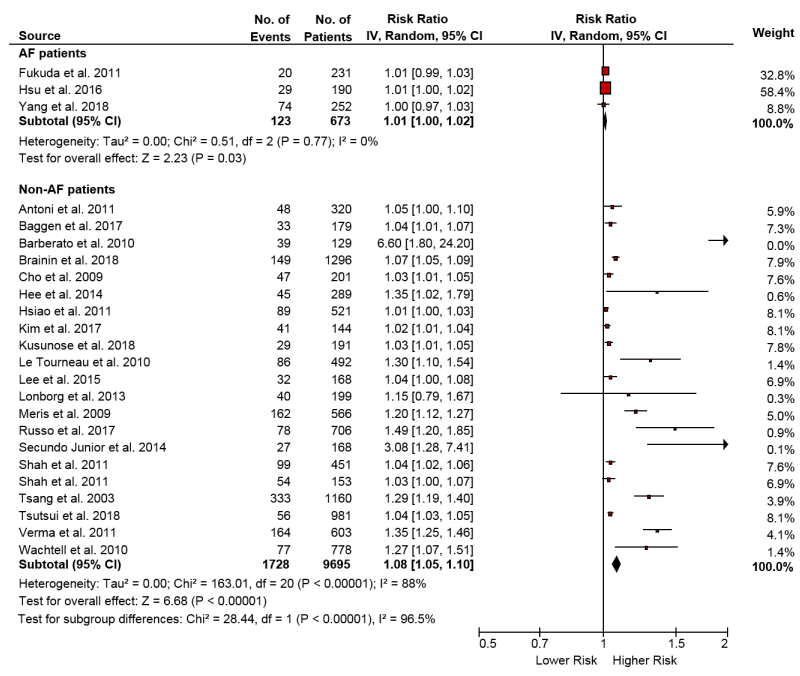

Figure 4 Association of LA volume index and risk of cardiovascular outcomes. Association of LA volume index with incident stroke or thromboembolic events (A), heart failure (B), all-cause mortality (C) and major adverse cardiac events (D). Data are risk ratios with corresponding 95\% Cls from inverse variance-weighted (IV) random-effects models. Meta-analysis results are separately presented as diamonds for studies including patients with $\mathrm{AF}$ and studies without patients with $\mathrm{AF}$. AF, atrial fibrillation; LA, left atrial.

process. For this meta-analysis, we did not systematically search for studies that reported LVDD parameters and cardiovascular outcomes. Whether these parameters substantially differ in patients with compared with patients without AF and whether they are differently associated with cardiovascular events is currently unclear and should be examined in future studies.

Increased LADI had a stronger association with stroke and cardiovascular outcomes than LAD. This may be explained by the fact that LADI is a more accurate measure of LA enlargement, leading to more accurate outcome prediction. However, the small number of studies using LADI as the predictor of interest lead to large confidence intervals around the effect estimates. On the other hand, the observed differences between LAV versus LAD measures, and outcome risk may be explained by two factors. First, while LA volume measures were more sensitive in predicting cardiovascular outcomes than LAD in previous studies, ${ }^{22} 23$ our analysis suggests the inverse. As most previous studies used calculated rather than directly measured LAV, this may have influenced the results. More studies are needed to address this issue. Second, only few studies provided information on absolute LAV measures and risk of outcome events. Thus, detailed comparison between LAV and LAVI was limited by the small number of studies available.

LA enlargement is a key risk factor for the development of $\mathrm{AF}^{824}$ and it is currently unclear how much of the associations in individuals without AF at baseline are due to intercurrent
AF development in some of these individuals. A previous study showed that among patients without AF with implanted devices, a significant proportion developed clinically unrecognised AF episodes. These usually short episodes were associated with an increased stroke risk, although the absolute risk was lower than in patients with clinical AF. ${ }^{25}$ While subclinical AF might be a mediator of the association between LA dimensions and stroke, it does not explain the stronger associations in non-AF populations.

A significant proportion of patients with AF may have been treated with oral anticoagulation (as shown in online supplementary tables S2 and S3), which is a highly effective treatment to reduce stroke in patients with $\mathrm{AF}^{26}$ and may have biassed the observed results in patients with AF towards the null. However, information on oral anticoagulation treatment was unavailable in many studies of patients without AF, which precluded more in-depth analyses on their influence on the observed risk differences.

Transthoracic echocardiographic imaging during AF episodes provides suboptimal visualisation of the atrium and may be complicated by irregular contraction of the left atrial wall. ${ }^{27}$ Thus, the reproducibility of measured LA parameters may be limited in patients with AF. Most studies that evaluated LA properties used 2D echocardiography technique, and volumes had to be calculated based on the method described by Simpson ${ }^{28}$ (see online supplementary tables S1 and S2). This technique is based 
on several assumptions and leads to significant measurement error. This may explain the finding that increased LAVI was not associated with a higher risk of stroke and thromboembolic events. A study using 3D echocardiography to evaluate LAV in individuals without a history of stroke and $<6 \%$ having a history of AF found that 3D measured LAV was independently associated with incident silent brain infarcts detected by brain MRI. ${ }^{29}$ Future studies should evaluate associations between LA parameters and adverse outcomes using 3D echocardiography. ${ }^{22} 30$

Heterogeneity was significant for most analyses. We conducted various subgroup analyses and found significant differences in effects across studies stratified by study design, geographical regions and publication years. In addition, meta-regression analyses did not indicate any effect of sample size, demographics or follow-up duration on the associations. We also did a sensitivity analyses by excluding studies of lower methodological quality, and observed similar findings. Nevertheless, it should be noted that residual sources of heterogeneity such as differences in patient characteristics, echocardiographic measurement methods or outcome definitions may have contributed to these findings.

\section{Strengths and limitations}

The strength of this meta-analysis is the use of accurate study methodology, including strict inclusion criteria for studies that only used a longitudinal design, comprehensive literature search strategy, in duplicate full-text review, sufficient data extraction and appropriate study quality assessment. However, there are some limitations that need to be taken into account. First, the reported associations were estimated based on aggregated studylevel rather than patient-level data. Thus, results should be interpreted cautiously in light of potential ecological bias. Second, we predefined inclusion criteria for studies that either had enrolled more than $90 \%$ (AF studies) or less than 10\% of patients with AF (non-AF studies). The inclusion decision was therefore based on population characteristics and reported methodology in the studies. Thus, we cannot exclude that some patients in non-AF studies may have had underdiagnosed AF. Third, endpoint definitions of MACE differed between the included studies. Fourth, LA dimension measures were either reported as continuous or categorical variable. Therefore, when pooling these data, analyses

\section{Key messages}

What is already known on this subject?

- Patients with left atrial (LA) enlargement have a higher risk for incident atrial fibrillation (AF), stroke and death, but studies including patients with AF not always confirmed these associations.

\section{What might this study add?}

- In this meta-analysis including 23 studies of patients with AF and 68 studies of patients without AF, LA echocardiographic parameters were positively associated with cardiovascular events in both patients with AF and without AF.

- Compared with patients with $A F$, many associations were stronger among patients without $\mathrm{AF}$, highlighting the potential importance of LA myopathy.

\section{How might this impact on clinical practice?}

- Future studies should address the question whether a LA echocardiographic screening should be implemented in patients without AF. had to be restricted to the relevant variable category. This may have led to a decrease in power. However, the observed trend that many associations were stronger among patients without AF was consistent throughout variable categories (see online supplementary figures S1-S4).

\section{CONCLUSIONS}

In this meta-analysis, LA size measured by transthoracic echocardiography was associated with an increased risk of several cardiovascular events. Of note, many of these associations were stronger among individuals without AF. This information may lend support to the concept of LA myopathy.

Correction notice Since this article was first published online, an equal contributor statement has been added for the first two authors.

Acknowledgements We thank Hannah Ewald, MPH (University Medical Library, University of Basel), for reviewing our search strategy.

Contributors The study concept and design was developed by LF, PM and DC. Search strategy and data acquisition was conducted by LF and PM. The statistical analyses were performed by LF and PM. LF, PM and DC drafted the manuscript. $L F, P M, S A, S B, D D, M K, S O, B A K$ and DC contributed to data interpretation and provided critical revision of the manuscript for important intellectual content. DC has been the study supervisor and is the corresponding author.

Funding The authors have not declared a specific grant for this research from any funding agency in the public, commercial or not-for-profit sectors.

Competing interests None declared.

Patient consent for publication Not required.

Provenance and peer review Not commissioned; externally peer reviewed.

Data availability statement All data relevant to the study are included in the article or uploaded as online supplementary information.

\section{ORCID iDs}

Pascal Meyre http://orcid.org/0000-0002-1236-1386

Stefanie Aeschbacher http://orcid.org/0000-0001-8134-2421

\section{REFERENCES}

1 Krijthe BP, Kunst A, Benjamin EJ, et al. Projections on the number of individuals with atrial fibrillation in the European Union, from 2000 to 2060. Eur Heart J 2013;34:2746-51.

2 Miyasaka Y, Barnes ME, Gersh BJ, et al. Secular trends in incidence of atrial fibrillation in Olmsted County, Minnesota, 1980 to 2000, and implications on the projections for future prevalence. Circulation 2006;114:119-25.

3 Conen D, Chae CU, Glynn RJ, et al. Risk of death and cardiovascular events in initially healthy women with new-onset atrial fibrillation. JAMA 2011;305:2080-7.

4 Wang $\mathrm{G}$, Joo $\mathrm{H}$, Tong $\mathrm{X}$, et al. Hospital costs associated with atrial fibrillation for patients with ischemic stroke aged 18-64 years in the United States. Stroke 2015;46:1314-20.

5 Wolf PA, Abbott RD, Kannel WB. Atrial fibrillation as an independent risk factor for stroke: the Framingham Study. Stroke 1991;22:983-8

6 Goette A, Kalman JM, Aguinaga L, et al. EHRA/HRS/APHRS/SOLAECE expert consensus on atrial cardiomyopathies: definition, characterization, and clinical implication. Heart Rhythm 2017;14:e3-40.

7 Casaclang-Verzosa G, Gersh BJ, Tsang TSM. Structural and functional remodeling of the left atrium: clinical and therapeutic implications for atrial fibrillation. J Am Coll Cardiol 2008;51:1-11.

8 Conen D, Glynn RJ, Sandhu RK, et al. Risk factors for incident atrial fibrillation with and without left atrial enlargement in women. Int J Cardio/ 2013;168:1894-9.

9 Benjamin EJ, D'Agostino RB, Belanger AJ, et al. Left atrial size and the risk of stroke and death. Circulation 1995;92:835-41.

10 Gupta DK, Giugliano RP, Ruff CT, et al. The prognostic significance of cardiac structure and function in atrial fibrillation: The ENGAGE AF-TIMI 48 Echocardiographic Substudy. J Am Soc Echocardiogr 2016;29:537-44.

11 Leung $M$, van Rosendael PJ, Abou R, et al. Left atrial function to identify patients with atrial fibrillation at high risk of stroke: new insights from a large registry. Eur Heart J 2018:39:1416-25.

12 Stroup DF, Berlin JA, Morton SC, et al. Meta-analysis of observational studies in epidemiology: a proposal for reporting. Meta-analysis Of Observational Studies in Epidemiology (MOOSE) group. JAMA 2000;283:2008-12.

13 Wells G, Shea B, O'Connell D. The Newcastle-Ottawa scale (NOS) for assessing the quality of nonrandomized studies in meta-analyses. Available: http://www.ohri.ca/ programs/clinical_epidemiology/oxford.asp [Accessed 23 May 2013]. 
14 Higgins J, Green S. Cochrane handbook for systematic reviews of interventions version 5.1.0. The Cochrane Collaboration, 2011.

15 DerSimonian R, Laird N. Meta-analysis in clinical trials. Control Clin Trials 1986;7:177-88.

16 Sterne JA, Egger M. Funnel plots for detecting bias in meta-analysis: guidelines on choice of axis. J Clin Epidemiol 2001;54:1046-55.

17 Higgins JPT, Thompson SG. Quantifying heterogeneity in a meta-analysis. Stat Med 2002:21:1539-58.

18 Guichard J-B, Nattel S. Atrial cardiomyopathy: a useful notion in cardiac disease management or a passing FAD? J Am Coll Cardiol 2017;70:756-65.

19 Watson T, Shantsila E, Lip GYH. Mechanisms of thrombogenesis in atrial fibrillation: Virchow's triad revisited. Lancet 2009;373:155-66.

20 Vaziri SM, Larson MG, Lauer MS, et al. Influence of blood pressure on left atrial size. The Framingham heart study. Hypertension 1995;25:1155-60.

21 Rusinaru D, Tribouilloy C, Grigioni F, et al. Left atrial size is a potent predictor of mortality in mitral regurgitation due to flail leaflets: results from a large international multicenter study. Circ Cardiovasc Imaging 2011:4:473-81.

22 Schaaf M, Andre P, Altman M, et al. Left atrial remodelling assessed by 2D and 3D echocardiography identifies paroxysmal atrial fibrillation. Eur Heart J Cardiovasc Imaging 2017;18:46-53.

23 Tsang TSM, Abhayaratna WP, Barnes ME, et al. Prediction of cardiovascular outcomes with left atrial size: is volume superior to area or diameter? J Am Coll Cardiol $2006 ; 47: 1018-23$.
24 Tsang TSM, Barnes ME, Bailey KR, et al. Left atrial volume: important risk marker of incident atrial fibrillation in 1655 older men and women. Mayo Clin Proc 2001;76:467-75.

25 Healey JS, Connolly SJ, Gold MR, et al. Subclinical atrial fibrillation and the risk of stroke. N Engl J Med 2012;366:120-9.

26 Hart RG, Pearce LA, Aguilar MI. Meta-analysis: antithrombotic therapy to prevent stroke in patients who have nonvalvular atrial fibrillation. Ann Intern Med 2007:146:857-67.

27 Kotecha D, Mohamed M, Shantsila E, et al. Is echocardiography valid and reproducible in patients with atrial fibrillation? A systematic review. Europace 2017:19:1427-38.

28 Lang RM, Bierig M, Devereux RB, et al. Recommendations for Chamber Quantification: a report from the American Society of Echocardiography's Guidelines and Standards Committee and the Chamber Quantification Writing Group, developed in conjunction with the European Association of Echocardiography, a branch of the European Society of Cardiology. J Am Soc Echocardiogr 2005; 18:1440-63.

29 Russo C, Jin Z, Liu R, et al. LA volumes and reservoir function are associated with subclinical cerebrovascular disease: the CABL (cardiovascular abnormalities and brain lesions) study. JACC Cardiovasc Imaging 2013;6:313-23.

30 Bossard M, Knecht S, Aeschbacher S, et al. Conventional versus 3-D echocardiography to predict arrhythmia recurrence after atrial fibrillation ablation. J Cardiovasc Electrophysiol 2017;28:651-8. 